\title{
Quantification of in-grain lattice gradient in neutron irradiated 304L SS during deformation using insituEBSD
}

Nitish Bibhanshu ${ }^{1}$, Maxim N. Gussev ${ }^{1}$ and Thomas M. Rosseel ${ }^{2}$

${ }^{1}$ Oak Ridge National Laboratory, Oak Ridge, Tennessee, United States, ${ }^{2}$ Oak Ridge National Laboratory, United States

Austenitic stainless steels (SS) with high fractions of annealing twin boundaries ( 43\%) are being used as structural components in nuclear reactors [1,2]. Exposure to irradiation causes elemental segregations, structural transformation, and dislocation loop formations, and, under an applied load, they form in-grain lattice gradients, deformation twins, and phase transformations [2,3]. This causes instability in materials and further failure. Although the literature has reported in-grain lattice gradient based on the color change in inverse pole figure (IPF), quantification techniques have not been well established [4,5]. We present an improved quantification method based on the pole figure and tensile direction (TD) IPF tringle.

The 304L SS with composition Fe-18Cr-10Ni-2Mn-0.5Si-0.25Cr-0.02C (weight \%) was selected for the present work. This alloy was subjected to neutron irradiation in the Russian BOR-60 fast reactor in the framework of the BORIS irradiation experiments conducted between 2000 and 2010. The 100 dpa irradiated sample was used to quantify the change in the in-grain lattice gradient. Quantification results were obtained using electron backscatter diffraction (EBSD) - generated data during in-situ tensile deformation inside a scanning electrion microscope (TESCAN MIRA3) equipped with a high-speed Oxford Symmetry EBSD system. EBSD data were recorded at $20 \mathrm{kV}$ and step sizes of $0.5 \square \mathrm{m}$.

EBSD is a powerful tool that is used to identify crystallographic orientation-dependent deformation mechanisms. The high-speed data acquisition of the EBSD detector during deformation made the quantification of changes possible, even at small deformation steps. Fig. 1 depicts image quality (IQ) and IPF maps at $0 \%$ and $10 \%$ of local strain. Local strain measurements are reported based on the average value of five length measurements between points noted in Fig. 1(a). At 10\% deformation, it shows channels of slip bands, with their direction marked by red arrows for the grains G1, G2, and G3 (Fig. 1(b)). The grain with less fraction of slip bands are showing a higher change in color gradient within it (Fig. 1(c) and Fig. 1(d)). Color change in the IPF map is caused by the local lattice gradient [4]. The grains G1, G2, and G3 after deformation are shown in Fig. 2(a), 2(b) and 2(c), respectively. The slip bands, consisting of channel dislocations and their arrangement, lead to the formation of low-angle grain boundaries (LAGb) and medium-angle grain boundaries (MAGb) as shown in Fig. 2(a) and 2(b). The higher fraction of the accumulated dislocations can be found near the high-angle grain boundary (HAGb) due to their pileup at the HAGb[6]. These dislocations along the HAGb lead to the formation of LAGb in the early stages of deformation. Furthermore, with an increase of deformation, these LAGb convert into the MAGb. Connectivity between the LAGb and MAGb is shown in Fig. 2(b). Crystallographic orientation for the grains G1, G2, and G3 at $0 \%$ and $10 \%$ are presented in a TD IPF tringle and shown in Fig. 2(d) and 2(f). Their (111) $\square$ pole figures at $0 \%$ and $10 \%$ are presented in Fig. 2(e) and 2(g), respectively. On deformation, spread in the pole intensities in the pole figures for G1, G2, and G3 are representing the formation of in-grain lattice gradient, and the same is identifiable in TD IPF map. This in-grain lattice gradient results mainly from geometrically necessary dislocations. In conclusion, the pole figures and IPF tringle represent the magnitude of in-lattice gradients for the grains G1, G2, and G3 as $10 \square, 7 \square$, and $11 \square$ tilt with respect to normal direction, respectively.

[This manuscript has been authored in part by UT-Battelle LLC under contract DE-AC0500OR22725 with the US Department of Energy (DOE). The US government retains and the publisher, by 
accepting the article for publication, acknowledges that the US government retains a nonexclusive, paidup, irrevocable, worldwide license to publish or reproduce the published form of this manuscript, or allow others to do so, for US government purposes. DOE will provide public access to these results of federally sponsored research in accordance with the DOE Public Access Plan (http://energy.gov/downloads/doepublic-access-plan).]
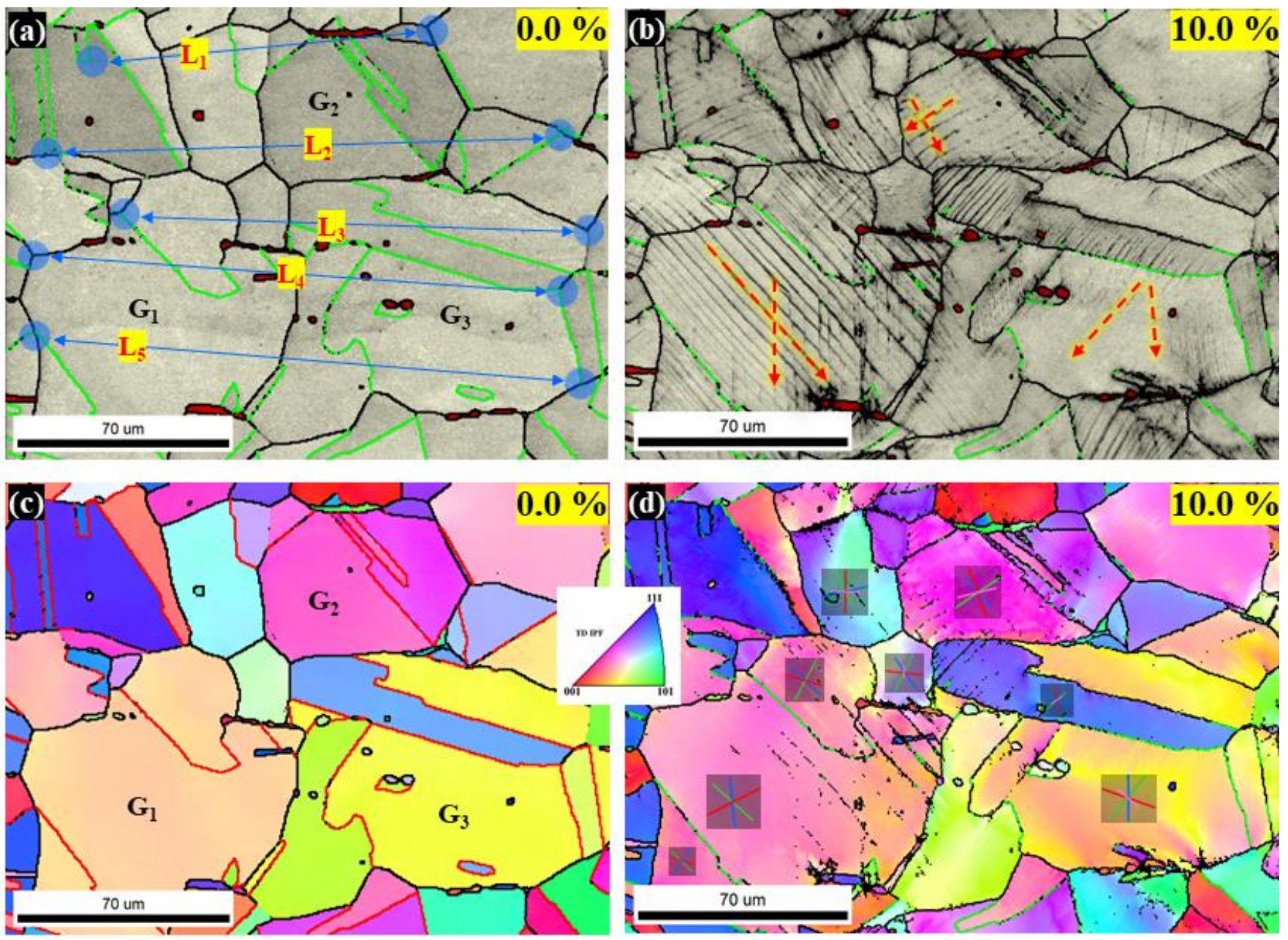

Figure 1. EBSD-generated IQ map at strain (a) $0 \%$ and (b) $10 \%$, and tensile direction IPF map at (c) $0 \%$ and (d) $10 \%$ of deformation 
(a)
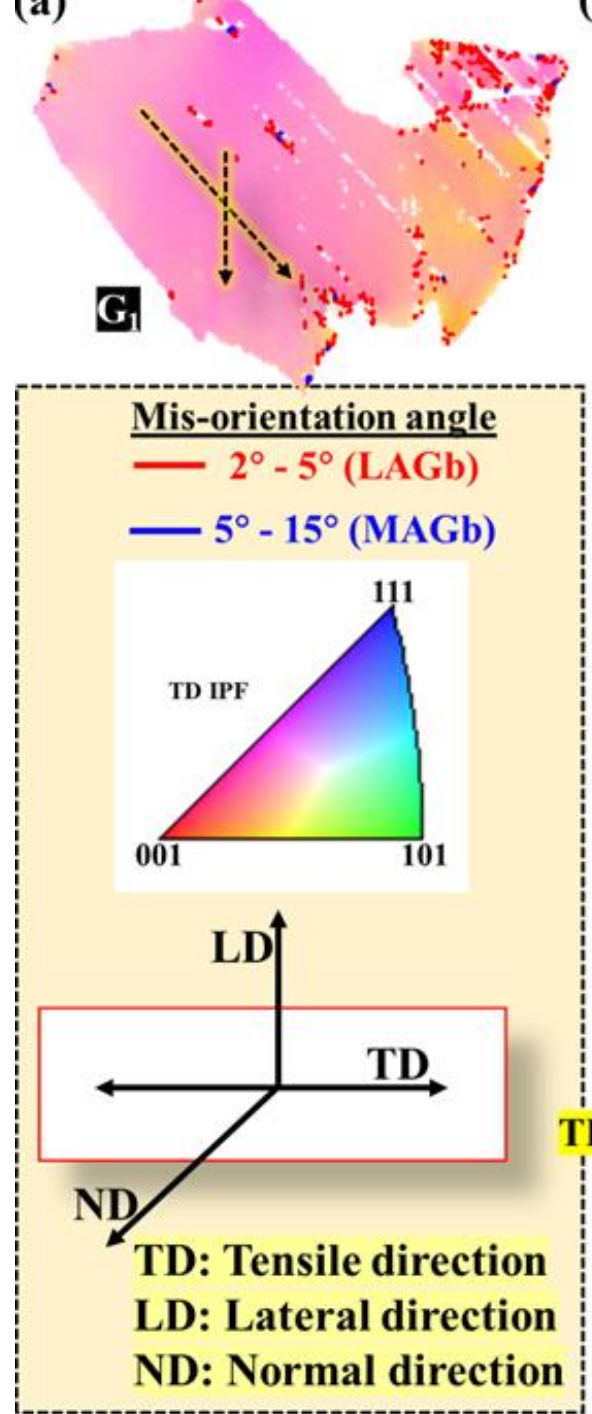

(b)

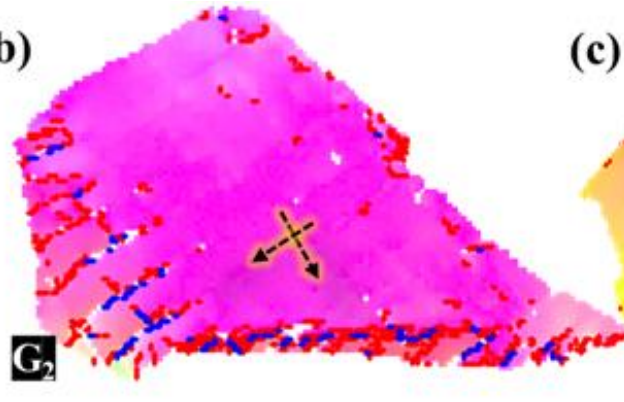

(d)

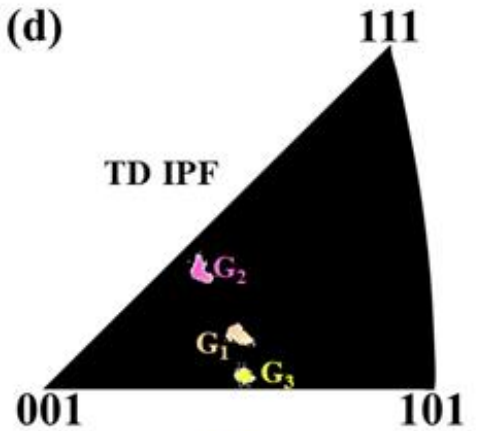

(f)

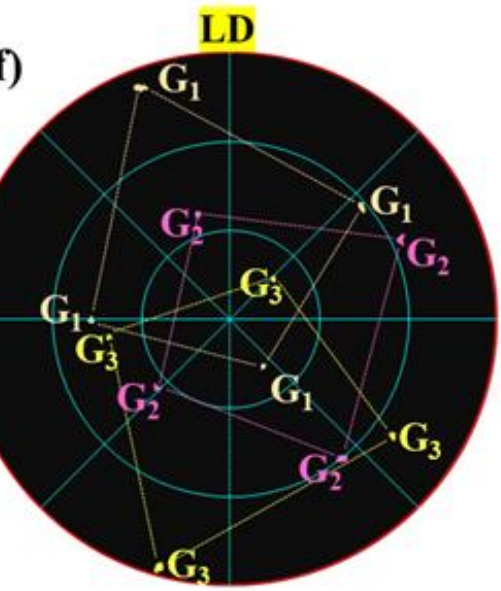

(c)

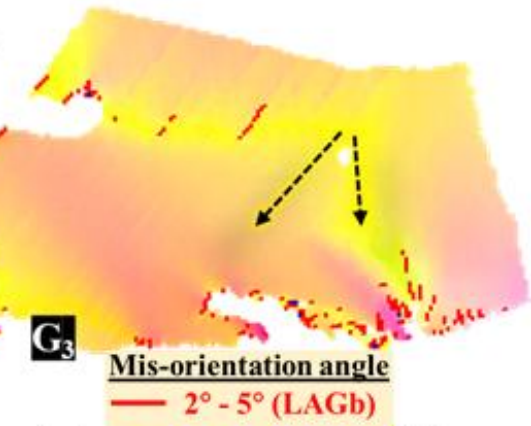

(e) $-5^{\circ}-15^{\circ}$ (MAGb) 11

Figure 2. Grain (a) G1, (b) G2, and (c) G3 at 10\%. Orientation of G1, G2, and G3 in TD IPF at strain (d) $0 \%$ and (e) $10 \%$ as well as in (111) $\gamma$ pole figures at (f) $0 \%$ and (g) $10 \%$.

\section{References}

[1] Carlander et al., Nuclear Applications and Technology 7 (1969) 67-75.

[2] Toyama et al., Journal of Nuclear Materials 418 (2011) 62-68.

[3] Dong et al., Journal of Nuclear Materials 467 (2015) 692-702.

[4] Bibhanshu et al., Journal of Materials Research 35 (2020) 1635-1646.

[5] Gussev et al., Journal of Nuclear Materials 517 (2019) 45-56.

[6] Wang et al., Philosophical Magazine 88 (2008) 1321-1343.

Acknowledgements: Present work was conducted under the aegis of the US Department of Energy, Office of Nuclear Energy, for the Light Water Reactor Systems program of research and development. We would like to thank Dr. Jason Harp (Oak Ridge National Laboratory [ORNL]), and Dr. Mikhail A. Sokolov (ORNL) for the technical review. Laurie Varma (ORNL) is gratefully acknowledged for the technical editing. We also thank T. Dixon (ORNL) for help with specimen preparation that was suitable for the insitu experiment. 